\title{
e-Migrinter
}

$22 \mid 2021$

Varia

\section{Transmisión, usos y representaciones lingüísticas de lenguas de herencia árabe y amazig entre estudiantes universitarios en Cataluña}

\section{Mònica Barrieras Angàs y Carla Ferrerós Pagès}

\section{(2) OpenEdition}

\section{Journals}

Edición electrónica

URL: https://journals.openedition.org/e-migrinter/2689

DOI: 10.4000/e-migrinter.2689

ISSN: 1961-9685

\section{Editor}

UMR 7301 - Migrinter

Referencia electrónica

Mònica Barrieras Angàs et Carla Ferrerós Pagès, « Transmisión, usos y representaciones lingüísticas de lenguas de herencia árabe y amazig entre estudiantes universitarios en Cataluña », e-Migrinter [En ligne], 22 | 2021, mis en ligne le 25 novembre 2021, consulté le 17 décembre 2021. URL : http:// journals.openedition.org/e-migrinter/2689; DOI : https://doi.org/10.4000/e-migrinter.2689

Este documento fue generado automáticamente el 17 diciembre 2021.

Tous droits réservés 


\title{
Transmisión, usos y
} representaciones lingüísticas de lenguas de herencia árabe y amazig entre estudiantes universitarios en Cataluña

\author{
Mònica Barrieras Angàs y Carla Ferrerós Pagès
}

\section{Introducción}

1 El presente artículo se incluye dentro de la investigación sobre lenguas de herencia, es decir, dentro de la investigación sobre lenguas de comunidades emigradas a otros estados. En este contexto se incluyen también estudios sobre lenguas indígenas, subordinadas o no oficiales en un estado determinado. Las investigaciones en este ámbito distinguen dos concepciones de las lenguas de herencia: la primera tiene en cuenta no solo las cuestiones lingüísticas sino también la herencia cultural vehiculada a través de la interacción familiar de los individuos emigrados (Van Deusen-Scholl, 2003). Esta definición hace referencia a individuos que, más que hablantes de una lengua de herencia, son aprendientes culturalmente motivados que, por lo tanto, tienen la lengua familiar como una segunda lengua (L2) (Polinsky y Kagan, 2007). La segunda definición del concepto de lengua de herencia, que es la que cuenta con más aceptación, la propone Valdés (2000): según esta autora, los hablantes de lenguas de herencia son individuos que han crecido en hogares donde se habla otra lengua que no es la del territorio de acogida. La lengua de herencia es la primera que aprenden, pero no es necesariamente del todo adquirida. La discusión se centra, pues, en la consideración de la lengua de herencia como L1 o L2. La diversidad de situaciones que podemos encontrar incluidas dentro de la categoría de lengua de herencia admite, según nuestro punto de vista, las dos posibilidades. Por otro lado, las lenguas de herencia han sido consideradas tradicionalmente como lenguas amenazadas, aunque, según destacan 
algunos autores, parece observarse cierto despertar de las lenguas de origen, a la vez que aparecen nuevas posibilidades de desafiar la homogeneización (Badosa, 2018).

2 En este artículo nos centramos exclusivamente en lenguas habladas por comunidades procedentes de otros estados en Cataluña. Analizaremos la transmisión de la lengua familiar (árabe y amazig) de individuos que han crecido en este territorio. Se han identificado más de 300 lenguas de todo el mundo habladas en Cataluña (GELA) ${ }^{1}$, debido al fenómeno migratorio vivido a comienzos del s. XXI. Es una migración relativamente reciente, pero ya han pasado suficientes años para que se pueda estudiar la transmisión intergeneracional de las lenguas de herencia en individuos que han nacido en Cataluña o que han llegado de pequeños o jóvenes.

3 En los últimos años se ha desarrollado la investigación de lo que se ha denominado family language policy (política lingüística familiar), que se centra en el estudio de la planificación producida en entornos familiares en relación a las lenguas y que analiza las prácticas lingüísticas, las ideologías $\mathrm{y}$, en caso de existir, las intervenciones y planificaciones que se hacen para modificar estas ideologías. El estudio sobre la planificación familiar es relevante en la investigación sobre lenguas de herencia, ya que la «family language policy approaches deepen our understanding of home language maintenance processes as well as how heritage language learners are best supported» (King, $2008,4)$. Nuestro análisis se basa también en ideologías o representaciones lingüísticas y se fija en los resultados de la planificación lingüística familiar en los hogares de los encuestados.

4 Los estudios sobre planificación lingüística familiar se relacionan con uno de los factores que intervienen en la política de la lengua: los denominados enfoques bottomup, contrapuestos a los top-down (Davis, 2014). El enfoque bottom-up tiene en cuenta los actores sociales que, por iniciativa propia, intervienen en la gestión lingüística:

[L]a PoL [política de la lengua] no solo es un instrumento que controla el estadonación sino que se elabora por parte de los individuos, las comunidades y en el seno de las familias a los que históricamente la investigación en PoL ha prestado muy poca atención, o por lo menos ha tardado en abordar. (Moustaoui Srhir, 2019, 181)

Estos procesos pueden tener un papel importante en la gestión de las lenguas en un entorno multilingüe y en contextos migratorios. En estos casos, el estudio de la planificación lingüística de las familias muestra las relaciones que se establecen en la gestión del multilingüismo entre el ámbito privado y el ámbito público (CurdChristiansen, 2013).

6 Algunas investigaciones hacen referencia a la imprevisibilidad en las prácticas lingüísticas de las familias alóglotas. La imprevisibilidad es una de las características enumeradas, junto con la movilidad y la complejidad, por Blommaert (2013). Por este motivo, algunos autores afirman que no es posible hablar de políticas lingüísticas familiares, ya que en general el término política lingüística se utiliza para hablar de contextos relacionados con las instituciones y la esfera pública (Bastardas-Boada, 2016). Así, más que hablar de políticas lingüísticas familiares, hay autores que afirman que se debe hablar de patrones de uso de las lenguas o de planificación lingüística (CurdChristiansen, 2009; Moroni, 2019).

7 En este artículo no nos centraremos en analizar cómo se producen estos procesos de planificación lingüística familiar (aspecto abordado por otros autores, como Moroni (2018), Shir (2018) o Fukuda (2020)). Este estudio es una primera aproximación a los resultados de la posible transmisión de familias alóglotas establecidas en Cataluña. A 
partir de un análisis cuantitativo de los datos, se analizará en qué grado se han transmitido el árabe y el amazig en un segmento de la población concreto: estudiantes alóglotos de la Universitat de Barcelona. En los casos en los que ha habido transmisión se analizarán los usos y las representaciones lingüísticas.

Este análisis tendrá en cuenta otro factor: el de la identificación con las lenguas propias (árabe y/o amazig) y las lenguas de acogida (catalán y/o español). Se pretende determinar si la situación sociolingüística catalana tiene alguna influencia en la transmisión de las lenguas de herencia, en particular cuando son minorizadas. Estudios anteriores (Junyent et al., 2011; Cortès et al., 2016; Barrieras y Ferrerós, 2019) han mostrado que, en algunos casos, se produce un efecto de empatía con la situación del catalán por parte de hablantes alóglotos de lenguas minorizadas que puede modificar las actitudes hacia las lenguas propias y, a la vez, hacia las lenguas de acogida, en este caso, el catalán.

9 Los datos utilizados para este estudio pertenecen, como hemos avanzado, a estudiantes de primera lengua (L1) árabe y/o amazig de la Universitat de Barcelona. Forman parte de un grupo con una serie de características particulares, ya que son jóvenes que han accedido a estudios universitarios. Los resultados, pues, no pueden hacerse extensivos a toda la población joven alóglota, tampoco a otro grupo parecido de otra universidad catalana. Aun así, los resultados que se obtengan en este estudio constituyen una aproximación útil a la transmisión de las lenguas de herencia en Catalunya.

10 Uno de los aspectos interesantes que presenta la comunidad marroquí es que permite estudiar dos lenguas de herencia diferentes que responden también a realidades lingüísticas diferentes: el árabe y el amazig. El árabe es oficial en el estado de origen. A pesar de que el árabe darija (la variedad hablada en Marruecos) se encuentra en una situación de diglosia respecto al árabe estándar moderno - el de la educación o los medios - o al árabe clásico -el de la religión (Albirini, 2016; Ennaji, 2005) - el hecho de que sus hablantes lo perciban como una variedad de una lengua que consideran prestigiosa hace que la percepción que puedan tener de ella sea diferente a la de los hablantes de amazig. El amazig, en cambio, es una lengua eminentemente oral - que se encuentra en una situación de triple diglosia en los territorios de origen - respecto al árabe darija y al árabe estándar o clásico, sin contar las lenguas de la colonización (Ennaji, 2005). A esta situación de diglosia, hay que añadir que la la falta de inteligibilidad provocada por la fragmentación dialectal hace que el amazig estándar que se propone y propaga a través de las escuelas y los medios esté alejado de la lengua hablada (Albirini, 2016; Ennaji, 2005). ${ }^{2}$

11 Así pues, los informantes de este estudio, a pesar de que proceden de la misma zona, parten de realidades lingüísticas muy diferenciadas. Partimos de la hipótesis de que estas diferencias repercutirán en la transmisión, los usos y las representaciones lingüísticas de dichos hablantes. Los objetivos de este estudio son, en primer lugar, analizar la transmisión de las lenguas de herencia árabe y amazig en Cataluña. A continuación, observar si hay diferencias en la transmisión del amazig respecto a la del árabe $\mathrm{y}$, en caso de que haya diferencias, si también se manifiestan en la lengua de identificación declarada por los hablantes. 


\section{Metodología}

12 En esta investigación se ha adoptado un enfoque mixto: el análisis es, sobre todo, cuantitativo, pero como la muestra relativamente reducida lo permite, va seguido de una fase cualitativa.

Los datos utilizados para el análisis se obtuvieron en el año 2015 a través de un cuestionario de preguntas semicerradas sobre usos y representaciones lingüísticas. Los criterios para la constitución de la muestra fueron intencionales: el cuestionario fue distribuido entre todos los estudiantes de la Universitat de Barcelona para que fuera respondido por aquellos que pudieran ser alóglotos o descendientes de familias que pudieran tener lenguas familiares distintas del catalán o del español.

14 Se obtuvieron 444 cuestionarios válidos una vez descartados los que no cumplían los siguientes criterios:

1. Estudiantes nacidos fuera del Estado español.

2. Estudiantes nacidos en territorios del Estado español con una lengua histórica diferente del catalán o del español, como por ejemplo el gallego o el vasco.

Se descartó a los descendientes de ciudadanos españoles nacidos en territorios hispanohablante No se eliminaron, en cambio, los nacidos ni los ascendientes de los nacidos en estados hispanoamericanos hablantes de lenguas americanas, ellos o sus ascendientes (Barrieras y Ferrerós, 2018). De esta muestra de 444 informantes, 34 eran estudiantes nacidos en Marruecos. Estos 34 cuestionarios son los que constituyen el corpus de este estudio. ${ }^{3}$

El cuestionario contenía numerosas preguntas, pero para el presente estudio se han seleccionado las que ofrecían datos que permitían alcanzar los objetivos que nos hemos propuesto. Así, se han tenido en cuenta las respuestas a las siguientes preguntas: la lengua hablada con la madre, la lengua hablada con el padre, la lengua inicial (es decir, la lengua en la que aprendieron a hablar), la lengua de identificación ${ }^{4}$ y la lengua utilizada con los hermanos. Una vez obtenidos los datos, se organizaron y etiquetaron mediante un procesador de datos y se analizaron cuantitativamente. Dado que trabajamos con un número relativamente reducido de datos (34 informantes), ha sido posible observarlas posteriormente con más detenimiento para poder hacer una descripción más detallada en el presente estudio.

\section{Resultados}

17 De una muestra de 444 informantes, 34 han nacido en Marruecos. De estos, podemos establecer tres grupos:

1. Grupo 1: 24 hablan árabe y no amazig

2. Grupo 2: 4 hablan amazig y árabe

3. Grupo 3: 6 hablan amazig pero no árabe

De los 34 informantes, 32 hablan más de una lengua (dejando de lado las consignadas) : en alguno de los campos del cuestionario declaran el francés, el catalán, el español, el inglés y el turco. Las dos últimas tienen una representación muy baja: un solo informante afirma que habla inglés y solo, a veces, con los hermanos. Otro informante 
declara el turco como lengua inicial ya que su padre nació en Turquía. A pesar de ello, en la pregunta sobre la lengua hablada con el padre, no declara el turco.

Hay 24 informantes que señalan que tienen el árabe como lengua inicial (grupo 1), tanto si la declaran como lengua única o lo hacen conjuntamente con otras lenguas. De ellos, 12 la tienen como lengua de identificación única y dos más se identifican con ella junto con el francés. 10 informantes es decir, menos de la mitad, se identifican con el francés, el español o el catalán, pero no con el árabe:

Tabla 1: lenguas de identificación de los hablantes de árabe (lengua inicial)

\begin{tabular}{|l|l|l|}
\hline Lengua inicial & \multicolumn{2}{|l|}{ Lengua de identificación } \\
\hline \multirow{1}{*}{ Árabe (24 informantes) } & 14 & $\begin{array}{l}\text { Árabe (12 informantes) } \\
\text { Árabe + francés (2 informantes) }\end{array}$ \\
\cline { 2 - 3 } & 10 & $\begin{array}{l}\text { Francés (2) } \\
\text { Español (5) } \\
\text { Catalán (2) } \\
\text { Catalán y español (1) }\end{array}$ \\
\hline
\end{tabular}

Si observamos los datos obtenidos de los hablantes de árabe, pero ahora teniendo en cuenta si declaran esta lengua como lengua inicial única o no, de los 24 informantes, 13 la declaran lengua inicial única. De estos 13, solo 6 se identifican con el árabe (uno de ellos junto con el francés) y 7 se identifican con otras lenguas, mayoritariamente con el español (5 informantes). Los otros 11 informantes declaran el árabe como lengua inicial junto con otras (el catalán, el castellano, el francés y, un informante, el turco). De estos 11, la mayoría (7 informantes) se identifican con el árabe y, uno de ellos, además, con el francés. Parece, pues, que el porcentaje de identificación con esta lengua es más alto para aquellos hablantes que declaran más de una lengua como lengua inicial.

Tabla 2: lengua de identificación según lengua inicial

\begin{tabular}{|l|l|l|}
\hline \multirow{2}{*}{ Lengua inicial } & \multicolumn{2}{|l|}{ Lengua de identificación } \\
\hline \multirow{2}{*}{ Árabe (13 hablantes) } & 5 & Árabe \\
\cline { 2 - 3 } & 1 & Árabe y francés \\
\cline { 2 - 3 } & 7 & $\begin{array}{l}\text { Otras lenguas } \\
\text { (castellano 5) }\end{array}$ \\
\hline $\begin{array}{l}\text { Árabe y otra lengua (11) } \\
\text { (catalán, castellano, francés, turco) }\end{array}$ & 7 & $\begin{array}{l}\text { Árabe } \\
\text { (francés 1) }\end{array}$ \\
\cline { 2 - 3 } & 4 & $\begin{array}{l}\text { Francés (2) } \\
\text { Catalán y español (2) }\end{array}$ \\
\hline
\end{tabular}

21 En cuanto a la lengua utilizada con los hermanos, dos de los 24 informantes declaran que hablan con ellos solo en árabe. Los dos se identifican con esta lengua y la han 
consignado como lengua inicial única. Encontramos, además, tres informantes que no utilizan esta lengua con ningún hermano y, en cambio, utilizan el catalán y/o el español, que son lenguas con las cuales se identifican. Los otros informantes sí utilizan el árabe con los hermanos junto con otras lenguas (habitualmente el catalán y el español, pero en algunos casos también el francés).

Así pues, en el caso del árabe, la transmisión es relativamente baja: 14 informantes se identifican únicamente con esta lengua (un poco más de la mitad), y solo dos la utilizan exclusivamente con los hermanos. Encontramos, además, tres informantes que no utilizan el árabe con los hermanos. En estos casos parecería, pues, que la transmisión de dicha lengua se ha interrumpido a favor del español y, en un caso, también del catalán. De los cuatro informantes que tienen el árabe y el amazig como lenguas iniciales (grupo 2), tres se identifican con el amazig y uno con ambas lenguas:

Tabla 3: lenguas de identificación de los hablantes de árabe y de amazig (lenguas iniciales)

\begin{tabular}{|l|l|l|}
\hline \multirow{2}{*}{ Lengua inicial } & \multicolumn{2}{|l|}{ Lengua de identificación } \\
\hline \multirow{2}{*}{ Árabe y amazig (4 hablantes) } & 3 & Amazig \\
\cline { 2 - 3 } & 1 & Amazig y árabe \\
\hline
\end{tabular}

De estos 4 informantes, 3 declaran que hablan con sus padres solo en amazig. Posiblemente añaden el árabe como lengua inicial, porque estuvieron expuestos a esta lengua de pequeños, aunque no fuera a través de sus progenitores. Estos informantes, además, son los que se identifican solo con el amazig y utilizan esta lengua para hablar con sus hermanos. El informante que queda, en cambio, sí utiliza las dos lenguas con sus padres. Se identifica con las dos y también las utiliza para hablar con sus hermanos. Por ello, en este grupo de hablantes de amazig y de árabe solo podríamos incluir de una manera clara a este último informante. Los otros tres, a pesar de afirmar que sus lenguas iniciales son las dos, podrían haberse incluido en el siguiente grupo.

El tercer grupo (grupo 3) está compuesto por 6 informantes que declaran el amazig como lengua inicial y, de estos, 4 se identifican con esta lengua. Otro lo hace con el español y uno con el catalán:

Tabla 4: lenguas de identificación de los hablantes de amazig (lengua inicial)

\begin{tabular}{|l|l|l|}
\hline \multirow{2}{*}{ Lengua inicial } & \multicolumn{2}{|l|}{ Lengua de identificación } \\
\hline \multirow{2}{*}{ Amazig (6 hablantes) } & 4 & Amazig \\
\cline { 2 - 3 } & 1 & Español \\
\cline { 2 - 3 } & 1 & Catalán \\
\hline
\end{tabular}

De estos 6 informantes que declaran el amazig como lengua inicial, 4 lo hacen como lengua única ( 3 de los informantes que se identifican con el amazig y el único informante que lo hace con el español). De los otros 2, uno declara como lenguas 
iniciales el amazig y el catalán (y es el que se identifica con esta lengua) y otro declara el amazig, el catalán y el español (y se identifica con el amazig). Este último afirma que casi no habla amazig con los hermanos, sino que utiliza el catalán, mientras que el resto se comunican con sus hermanos sobre todo en amazig.

27 Así pues, entre los seis hablantes de amazig que declaran esta lengua como inicial (junto con otras o no) hay un alto grado de identificación. A pesar de que se identifica con esta lengua, solo uno no habla amazig con sus hermanos, sino que utiliza el catalán. A estos seis hablantes, les podríamos sumar los tres informantes que hemos clasificado como hablantes de las dos lenguas, pero que tienen familias amazigófonas (del grupo 2). Teniendo en cuenta esto, podemos determinar que la identificación y transmisión de esta lengua es alta en nuestra muestra en comparación con el árabe.

A continuación, mostramos un resumen de los datos que hemos expuesto mediante gráficos:

Gráfico 1: Lengua de identificación de los hablantes de árabe como lengua inicial (GRUPO 1)

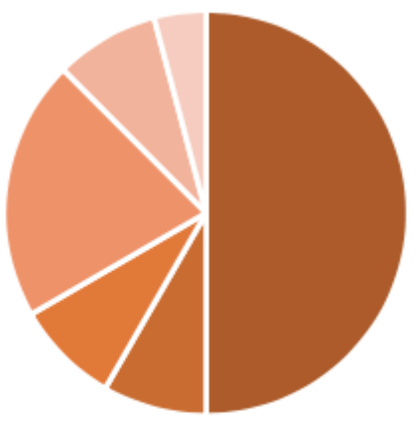

- Árabe "Árabe y francés " Francés "Español "Catalán "Españoly catalán

Gráfico 2: Lengua de identificación de los hablantes de árabe y amazig como lenguas iniciales (GRUPO 2)

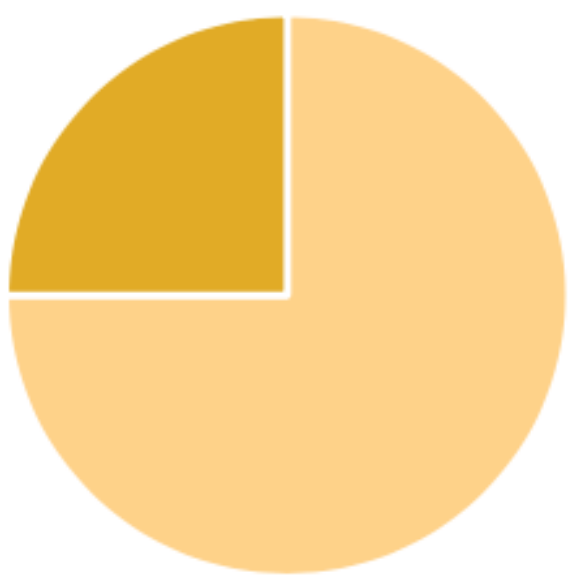

Amazig " Árabe y amazig 


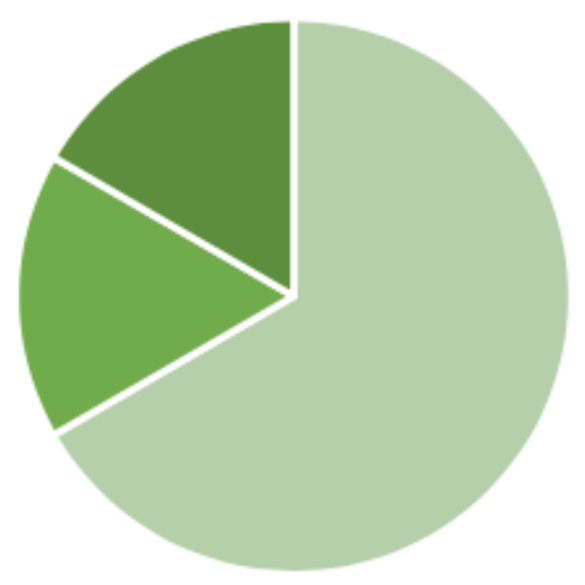

\section{- Amazig " Español " Catalán}

Hemos visto que la mitad de los informantes que tienen el árabe como lengua inicial se identifican únicamente con esta lengua (12 de 24 hablantes). Dos más se identifican con el árabe junto con el francés. En el caso de que se identifiquen con una de las lenguas de acogida, tiende a ser el español (5 informantes); dos lo hacen con el catalán, dos con el francés y uno con el catalán y el español. La mayor parte de informantes que tienen el árabe y el amazig como lenguas iniciales se identifica con el amazig (3 de 4). El otro informante lo hace con el amazig y el árabe. De los que declaran el amazig como lengua inicial única, la mayoría se identifica con ella (4 de 6). De los dos hablantes que no lo hacen, uno se identifica con el catalán y el otro con el español. Si sumamos los 3 informantes incluidos en el grupo 2, pero que parece que tienen familias amazigófonas, tendríamos los datos de identificación siguientes: ${ }^{5}$

- Hablantes de árabe (L1): 24 informantes, de los cuales 14 se identifican con el árabe (58,3\%)

- Hablantes de árabe y de amazig (L1): 1 informante que se identifica con ambas lenguas.

- Hablantes de amazig (L1): 9 informantes, 7 se identifican con el amazig (77,7 \%).

En lo que hace referencia a las lenguas habladas con los hermanos, dato importante que sugiere si ha habido transmisión de la lengua o no, hemos extraído los gráficos siguientes: 
Gráfico 4: lengua hablada con los hermanos (GRUPO 1)

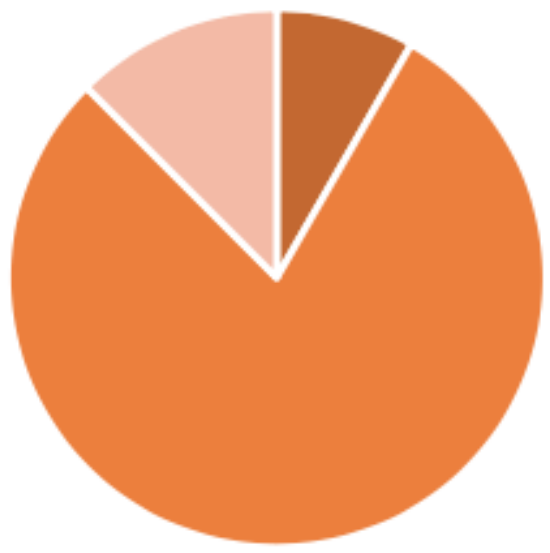

- Árabe " Árabe y otras "Otras

Gráfico 5: Lengua hablada con los hermanos (GRUPO 2)

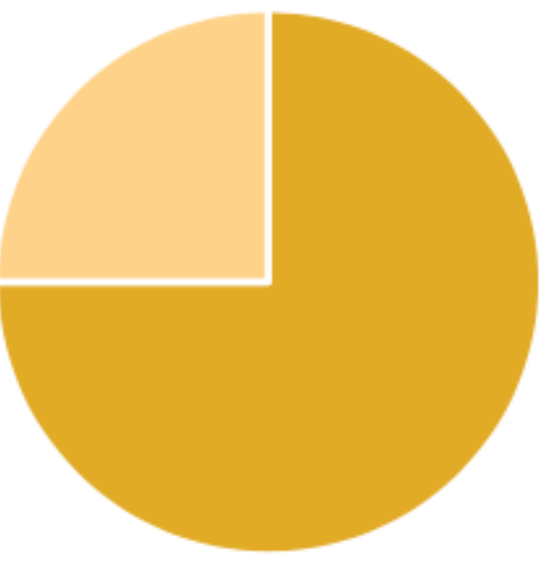

" Amazig = Árabe y amazig 


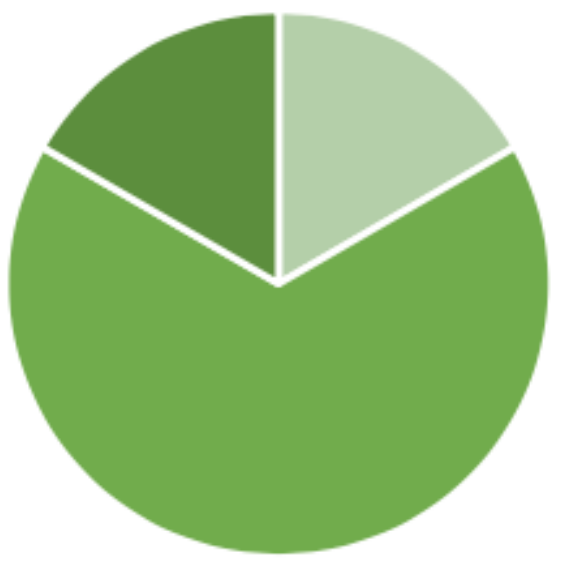

\section{- Amazig - Amazig y catalán " Español}

Todos los hablantes de amazig, excepto uno (grupo 2 y grupo 3) utilizan esta lengua con sus hermanos (exclusivamente o no). En cambio, encontramos tres hablantes arabófonos que no utilizan esta lengua con los hermanos: dos hablan con ellos en español y uno en catalán y en español.

\section{Discusión}

De una muestra de 444 hablantes alóglotos, solo 34 nacieron en Marruecos. Cataluña, tiene actualmente una población de 7555 830, y un 13,78 de la población es de origen extranjero, es decir, 1041362 personas (IDESCAT). Según datos del año 2017, en Cataluña residen 207082 ciudadanos de origen marroquí, que representan el 19,89\% de la población extranjera total y un 2,74 \% de la población total. El año 2000 habían llegado a representar el 33,46 \% de la población extranjera y el año 2012 el 3,16 \% de la población total: siempre ha sido la comunidad extranjera más numerosa en Cataluña. A pesar de esto, si se compara con otras comunidades establecidas en Cataluña, como la ucraniana, la marroquí está infrarrepresentada en la Universitat de Barcelona:

Tabla 5: representación de las comunidades marroquí y ucraniana sobre el total de extranjeros en Catalunya y sobre el número de estudiantes de la Universitat de Barcelona

\begin{tabular}{|l|l|l|}
\hline & Población total extranjera & UB \\
\hline Marroquíes & $19,89 \%$ & $7,6 \%$ \\
\hline Ucranianos & $2,05 \%$ & $2,25 \%$ \\
\hline
\end{tabular}

Esta infrarrepresentación ya ha sido mencionada en investigaciones anteriores. Serra (2010), por ejemplo, afirma que a pesar de que existen pocas investigaciones sobre la relación en Cataluña entre las personas nacidas fuera de la Unión Europea y el sistema educativo, parece que " los resultados escolares de los alumnos que proceden de la inmigración extracomunitaria obtienen resultados significativamente más bajos que los 
resultados que obtienen los alumnos autóctonos » (Serra, 2010, 29). ${ }^{6}$ Por este motivo, hay menos estudiantes de fuera de la Unión Europea que cursen estudios superiores.

Un estudio de 2018 sobre la relación entre la inmigración y el éxito escolar muestra que aquellos alumnos que han nacido fuera de Catalunya y que no se han incorporado al sistema educativo catalán desde el inicio muestran porcentajes altos de fracaso escolar:

[E]l fracaso sería menor entre los de origen europeo (11,3\%) y americano (15,5\%), donde la condición socioeconómica de los primeros y la proximidad con el idioma de los segundos justificarían los mejores resultados. Para el conglomerado asiático el fracaso asciende al 17,8 \% [...]. En la cola, con el 19,2\% entre los alumnos, encontramos a los de origen africano. En este último caso, el 15,9\% de fracaso escolar entre los alumnos marroquíes es preocupante tratándose del primer origen, especialmente si tenemos en cuenta el crecimiento de este grupo en un futuro inmediato. (Bayona i Domingo, 2018, 11)

La infrarepresentación de los alumnos de origen marroquí en la universidad, pues, explica la muestra reducida de la que hemos partido en este trabajo. A pesar del bajo número de participantes, los datos descritos permiten analizar la transmisión de las lenguas de herencia árabe y amazig. En un estudio anterior (Barrieras y Ferrerós, 2019) sobre todos los estudiantes alóglotos de la misma universidad (444 informantes) y no solo los de origen marroquí, los datos revelan que las lenguas minorizadas muestran un porcentaje de sustitución igual que el de las lenguas con más hablantes (alrededor del $11 \%)$ : es decir, no se observan diferencias entre lenguas minorizadas y lenguas dominantes en cuanto a interrupción de la transmisión intergeneracional. Así, los datos globales sugieren que la transmisión de todas las lenguas, y particularmente de las minorizadas, es más elevada de lo esperable, dada la dificultad de mantener la lengua de herencia bajo la presión social e institucional en favor de la lengua de acogida. Según Wong Fillmore (2000), pocas comunidades de inmigrantes han conseguido mantener sus lenguas cuando se han asimilado a la vida de la sociedad de acogida, en este caso la americana. La pérdida solía producirse en la segunda o tercera generación, mientras que actualmente el aprendizaje del inglés en la escuela es un modelo sustractivo, de manera que el inglés ya reemplaza la lengua familiar en los jóvenes de la primera generación (un proceso que se ha acelerado en los últimos 25 años). Brown considera que " the persistent loss of HL has been an historical phenomenon in the US, something both expected and accepted under the notion of a "melting pot" of many cultures" (2011:31). También describe las expectativas claras y explícitas en los Estados Unidos de que los immigrantes aprendan inglés durante su escolarización y pierdan su lengua de origen al mismo tiempo (Brown, 2000).

36 A pesar de lo relacionado en los estudios que acabamos de describir, en lo que hace referencia a los estudiantes de origen marroquí analizados en este estudio, podemos concluir que están en consonancia con la tendencia de la muestra del estudio global: la mayoría de los informantes habla sus lenguas familiares con los hermanos (9 de cada diez hablantes de amazig y 21 de 24 hablantes de árabe). Los porcentajes, pues, se sitúan alrededor del $90 \%$, en consonancia con los datos globales que hemos mencionado (el $89 \%$, puesto que el porcentaje de sustitución es del $11 \%$ ). En algunos casos, el uso que hacen de estas lenguas con los hermanos no es exclusivo y utilizan también las lenguas del territorio de acogida, el catalán o el español.

En cuanto a la lengua de identificación, el amazig lo es con más frecuencia que el árabe. Si tenemos en cuenta los informantes que tienen solo el amazig como lengua inicial, observamos en el $77 \%$ de los informantes del corpus se identifica con esta lengua. En 
cambio, si tomamos los informantes que consignan el árabe como lengua inicial, el 58,3 $\%$ se identifica con esta. En el caso de que no haya habido identificación con la lengua de herencia, en el conjunto del corpus encontramos tres situaciones: sustitución por el español, por el catalán o por la dominante en el territorio de origen (Barrieras y Ferrerós, 2019). Es destacable que con los hablantes de origen marroquí no encontramos sustitución del amazig por el árabe, es decir, no se produce la sustitución por la lengua dominante del territorio de origen. En cambio, sí se produce la sustitución por las lenguas de acogida. En este caso, se observa cierta tendencia hacia el catalán por parte de hablantes de amazig y hacia el español por parte de hablantes de árabe.

Estos resultados pueden relacionarse con otro fenómeno descrito, el llamado efecto espejo según el cual el contacto con la situación sociolingüística catalana puede actuar como un estímulo para algunas personas de origen extranjero, especialmente entre hablantes de lenguas minorizadas, para reevaluar su propia herencia cultural y lingüística o incluso reinterpretar sus orígenes lingüísticos (Cortès-Colomé et al.: 2016, 276). Se produce, pues, un efecto de empatía y reflejo por parte de los recién llegados que cambia las actitudes lingüísticas hacia la propia lengua y la diversidad lingüística en general, y a la vez comporta una adhesión a la lengua catalana (Junyent et al., 2011; Cortès et al., 2016). En entrevistas realizadas para estos estudios algunos hablantes expresan las siguientes ideas:

Yo creo que tuve este interés por mi lengua, por estudiar -sí tenía interés por estudiar, pero este interés más fuerte- después de hacer el nivel intermedio de catalán [...]. Cuanto más catalán hablo, más tártaro estoy. Tengo esta sensación. Que ahora tengo que estudiar tártaro. (Junyent et al., 2011, 99)

Descubrí sobre todo las semejanzas que hay entre el catalán y el amazig, y esto me ayudó a tener clara la realidad de Catalunya y lo que debo hacer respecto a esta realidad y la actitud que debo tener con mis hijos y el entorno [...] Una cosa refuerza la otra, para decirlo de algún modo. Descubrir el hecho diferencial catalán refuerza en mi la voluntad de luchar por el hecho diferencial amazig. (Junyent et al., 2011, 99)

Badosa (2018), en un estudio sobre el efecto espejo en individuos de lengua quechua y amazig, remarca que este efecto se produce en mayor proporción entre hablantes de amazig que entre hablantes de quechua. El autor distingue dos perfiles dentro de la comunidad amazig: el de las personas que ya eran sensibles a su herencia lingüística y el de las que desarrollaron esta sensibilidad ya en Cataluña. En el primer caso, los entrevistados empezaron a identificarse como amazigs cuando iniciaron sus estudios en universidades marroquíes y emigraron desde zonas rurales amazigs a ciudades donde el árabe era la lengua dominante. Una vez en Cataluña, el efecto espejo los ha llevado a sentirse amazig-catalanes, a usar el catalán como lengua prioritaria y a identificarse con ella. En el segundo caso, encontramos amazigs que llegaron a Cataluña de pequeños. En Cataluña, gracias al contacto con otros amazigs y con la situación catalana, se han convertido en defensores de la lengua y la cultura amazig, y usan el catalán como lengua preferida.

Estudios relacionados con las lenguas en secundaria en Cataluña muestran ideas que concuerdan con el efecto espejo. En el siguiente fragmento, extraído de Bretxa et al. (2017), un tutor de aula de acogida hace referencia al acercamiento al catalán de los alumnos amazigs:

Los alumnos que tienen el amazig como lengua materna tienen una situación parecida a la nuestra [entre el amazig y el árabe], y esto ha provocado una mejor predisposición, cierta simpatía, por aprender la lengua y utilizarla. [...] Actitud 
positiva y simpatía hacia el catalán, porque relacionan la situación con el amazig. (Manlleu, tutor de aula de acogida). (Bretxa et al. 2017, 106) ${ }^{8}$

41 Por otro lado, la procedencia de las familias alóglotas puede tener relación con la transmisión de las lenguas familiares. Moustaoui Shrir (2019) menciona, por ejemplo, cómo las desigualdades sociales y económicas pueden tener repercusiones en la planificación y en las actitudes lingüísticas de los marroquíes, y como el estatus de las lenguas de origen está relacionado con la transmisión:

En investigaciones anteriores, vi como la estratificación social, lingüística y laboral que afecta, más que a otras comunidades de trabajadores inmigrantes, a la comunidad marroquí, ha provocado la aparición de discursos de resistencia apoyados también en el mantenimiento de las lenguas de origen en el seno de estas comunidades. La utilización de lenguas no normativas como el árabe marroquí o estándar y el amazig en determinados contextos se considera como una práctica de contestación a las diferentes formas de asimilación; y la socialización familiar en las lenguas propias, como una forma de resistencia a dichas presiones. (Moustaoui Srhir, 2019, 184-185)

42 Así pues, uno de los fenómenos que explica el mantenimiento de las lenguas de herencia que hemos observado a partir de los datos analizados puede ser, precisamente, el de hablar lenguas sin estatus de oficialidad en el territorio de origen (árabe darija y amazig). No obstante, y como ya hemos avanzado, los datos obtenidos con la comunidad marroquí en este estudio no son sustancialmente distintos de los datos globales obtenidos para el conjunto del corpus, independientemente de cuál o cuáles fueran las lenguas de herencia de los alumnos encuestados (Barrieras y Ferrerós, 2019). La circunstancia de que el corpus está compuesto exclusivamente por estudiantes universitarios puede tener también alguna influencia en la naturaleza de los resultados.

Como hemos avanzado, los resultados de nuestro estudio sugieren que la realidad sociolingüística catalana puede ejercer cierta influencia en el mantenimiento de las lenguas de origen, especialmente para los alumnos que hablan lenguas minorizadas como el amazig. En este caso concreto, en Cataluña ha habido un interés y una atención especial hacia esta lengua y sus hablantes, hecho que también ha podido influir en las actitudes de los hablantes amazigófonos. ${ }^{9}$ Según diversas estimaciones (a falta de datos oficiales), la mayor parte de la población marroquí en Cataluña es de origen amazig y se encuentra a menudo concentrada en ciertas zonas, como la de Vic-Manlleu o la de Mataró, donde a veces hasta el 90 \% de los marroquíes son amazigs (Tilmatine, 2005). Se trataría del colectivo más numeroso de personas de origen extranjero con aproximadamente unas 180000 personas (Castellanos, Akioud, 2013). Se ha detectado, no obstante, cierta ocultación por parte de los hablantes de la lengua, a pesar de la presencia que ha tenido el amazig en Catalunya, por lo cual la cifra de hablantes podría ser incluso superior.

\section{Conclusiones}

44 A pesar de que la muestra es pequeña (ya que, como avanzáramos, hay pocos estudiantes de origen marroquí en la Universitat de Barcelona), una vez descritos y analizados los datos, podemos determinar que las lenguas de herencia árabe y amazig son transmitidas en porcentajes elevados, en consonancia con lo que habíamos observado en estudios previos y con otras lenguas de herencia (Barrieras y Ferrerós, 
2019). Esta tendencia contrasta con otros estudios que muestran que las lenguas de las comunidades emigradas suelen sustituirse por la lengua de acogida (Wong Fillmore, 2000; Brown, 2011). Teniendo en cuenta esto, habíamos afirmado en estudios previos que « los datos de nuestra muestra pueden estar condicionados por la circunscripción al ámbito universitario, entendiendo que la situación sociocultural podría tener cierta influencia en la transmisión de las lenguas de herencia » (Barrieras i Ferrerós, 2019, 243). ${ }^{10}$ Aun así, este factor no es claro: como mencionamos en el apartado anterior, Moustaoui Shrir (2019) ha observado que la resistencia a fenómenos de asimilación cultural y lingüística puede estar condicionada, precisamente, por la estratificación social y laboral de las familias de origen marroquí. Es posible que la explicación para el mantenimiento de estas lenguas de herencia tenga que ver más bien con el territorio de acogida: tanto Wong Fillmore (2000) como Brown (2011) contextualizan sus investigaciones en Estados Unidos.

En cuanto a los datos sobre identificación, también encontramos porcentajes elevados, aunque un poco menos que los de transmisión. En este caso, el amazig se consigna con más frecuencia que el árabe como lengua de identificación. Una posible explicación de este hecho es el efecto de reflejo con la situación sociolingüística catalana. El hecho de que el catalán, que no es una lengua mayoritaria, tenga presencia en ámbitos normalmente reservados a lenguas dominantes (medios de comunicación, escuela, administración) puede cambiar las actitudes lingüísticas y los comportamientos de los hablantes de otras lenguas minorizadas (Junyent et al., 2011; Cortès et al., 2016). Así, el efecto de empatía puede llevar a hablantes de lenguas minorizadas a profundizar, no solo en la lengua de acogida, sino en su propia lengua. Convendría determinar, en futuras investigaciones, si esta tendencia se mantiene o no en territorios de acogida monolingües.

Cuando los hablantes de lengua de herencia árabe o amazig se identifican con una lengua que no es la inicial, lo hacen sobre todo con el español (y menos con el catalán). Esto se contrapone a lo que hemos mencionado respecto al efecto espejo. Hay que señalar, no obstante, que aquellos que se identifican con el español son casi todos hablantes de árabe como L1, que es también una lengua dominante en relación al amazig. En cambio, no encontramos casos de sustitución de lenguas minorizadas habladas como L1 por la lengua dominante en el territorio de origen (del amazig por el árabe o incluso del árabe darija por el árabe estándar), excepto en el caso de dos personas arabófonas que se identifican con el francés, antigua lengua colonial de Marruecos. Este es el único dato que contrasta con los obtenidos del conjunto de la muestra, en los que sí hay sustituciones de este tipo. En cuanto a los hablantes de árabe darija, posiblemente no se da esta sustitución, porque el estándar no es lengua familiar de ningún grupo de marroquíes. En el caso del amazig, podría ser resultado de un conjunto de factores: una forma de resistencia, como explica Moustaoui Shrir (2019) ; el producto del efecto espejo, o el hecho de que los informantes de la muestra formen parte del ámbito universitario. 


\section{BIBLIOGRAFÍA}

Albirini, Abdulkafi (2016) Modern Arabic sociolinguistics: Diglossia, variation, codeswitching, attitudes and identity, Londres: Routledge, $438 \mathrm{p}$.

Badosa, Albert (2918) The mirror effect: languages ideologies of the amazigh and quechua communities in Catalonia, Tesis de máster, SOAS, University of London.

Badosa, Albert ; Ferrerós, Carla (2019) El poble amazic a Catalunya: aspectes d'actualitat. El poble amazic a Catalunya: contactes històrics, in El poble amazic a Catalunya, Barcelona: Ciemen, Nationalia, pp. 10-14.

Barrieras, Mònica ; Ferrerós, Carla (2019) Llengües minoritàries al-loglotes a Catalunya: una mostra d'estudiants universitaris, in Barrieras, Mònica i Ferrerós, Carla (eds.) Transmissions. Estudis sobre transmissió lingüística, Vic: Eumo, pp. 227-247.

Bastardas-Boada, Albert (2016) Famílies lingüísticament mixtes a Catalunya: competències, usos i autoorganització evolutiva, Treballs de sociolingüística catalana, Lleida: Pagès, pp. 89-116.

Bayona, Jordi ; Domingo, Andreu (2018) El fracàs escolar dels descendents de la immigració a Catalunya: més que una assignatura pendent, Perspectives demogràfiques, ${ }^{\circ}{ }^{\circ} 11$, pp. 1-4.

Blommaert, Jan (2013) Ethnography, Superdiversity and Linguistic Landscapes: Chronicles of Complexity, Bristol: Multilingual Matters, 144 p.

Bretxa, Vanessa ; Comajoan-Colomé, Llorenç ; Vila, Francesc Xavier (2017) Les veus del professorat. L'ensenyament $\mathrm{i}$ la gestió de les llengües a secundària, Barcelona, Horsori, $162 \mathrm{p}$.

Castellanos, Carles (2019) El poble amazic a Catalunya: contactes històrics, El poble amazic a Catalunya, Barcelona, Ciemen, Nationalia, pp. 5-9.

Chaker, Salem (1995) Dialecte. Vol. XV, Encyclopedie berbere, Aix-en- Provence, Edisud.

Brown, Clara Lee (2011) Maintaining Heritage Language: Perspectives of Korean Parents, Multilingual Education, vol.19, n¹, pp. 31-37.

Castellanos, Eva ; Akioud, Hassan (2013) Amazics: identitat i llengua a l'Àfrica del nord, DivÈrsia, $n^{\circ} 3$.

Cortès, Montserrat ; Barrieras, Mònica ; Comellas, Pere (2016) Changes in immigrant individuals' language attitudes through contact with Catalan: the mirror effect, Language Awareness, $\mathrm{n}^{\circ} 25, \mathrm{pp}$. 272-289.

Curd-Christiansen, Xiao Lan (2013) Negotiating Family Language Policy: Doing Homework, in Shwarz, M. i Verschik, A. (eds.) Successful Family Language Policy. Parents, Children and Educators in Interaction, Nova York: Springer, pp. 277-295.

Curd-Christiansen, Xiao Lan (2009) Invisible and visible language Planning: ideological factors in the family language policy of Chinese immigrant famílies in Quebec, Language Policy, $\mathrm{n}^{\circ} 8, \mathrm{pp}$.

351-357.

Davis, Kathryn (2014) Engaged language policy and practice, Language policy, vol.13, $\mathrm{n}^{\circ} 4$, pp. 83-100.

Ennaji, Moha (2005) Multilingualism, cultural identity, and education in Morocco, New York: Springer Science \& Business Media, $254 \mathrm{p}$. 
Fabà, A. ; Torres-Pla, J. (2020) De la llengua inicial a la llengua d'identificació a Catalunya: qui adopta el català i qui se n'allunya, Treballs de Sociolingüística Catalana, n³0, pp. 213-232.

Fukuda, Makiko (2020) Trajectòries lingüístiques dels progenitors japonesos de les famílies transnacionals multilingües a Catalunya, Treballs de Sociolingüística catalana, n³0, pp. 57-74.

GELA, Grup d'Estudi de Llengües Amenaçades: www.gela.cat

IDESCAT. Institut d'Estadística de Catalunya: https://www.idescat.cat/.

Junyent, Maria Carme ; Monrós, Eva ; Fidalgo, Mònica ; Cortès, Montserrat ; Comellas, Pere ; Barrieras, Mònica (2011) Canvi de representacions lingüístiques dels parlants al-loglots per contacte amb la situació sociolingüística catalana, Recerca i immigració III (convocatòria d'ajuts 2007), Collecció Ciutadania i Immigració n6, Generalitat de Catalunya, pp. 93-109.

Kossmann, Marteen (2012) Berber, in Frajzyngier, Z. i Shay, E. (eds.) The Afroasiatic languages, Cambridge, Cambridge University Press, pp. 18-101.

Moroni, Andrea (2018) Portugues como língua de herança na Catalunha: Superdiversidade, autoorganizaçao evolutiva e imprevisibilidade, in Barrieras, Mònica i Ferrerós, Carla (eds.) Transmissions. Estudis sobre transmissió lingüística, Vic, Eumo, pp. 211-226.

Moustaoui Shrir, Adil (2018) Política lingüística en familias transnacionales de origen marroquí: ideologies, prácticas y desafío, in Barrieras, Mònica i Ferrerós, Carla (eds.) Transmissions. Estudis sobre transmissió lingüistica, Vic, Eumo, pp. 179-210.

Múrcia, Carles (2015) Compendi gramatical, in Múrcia, C. i Zenia, S. (eds.) Diccionari català-amazic, amazic-català, Barcelona, Llibres de l'índex.

Naït-Zerrad, Kamal (2001) Esquisse d'une classification linguistique des parlers berbères, AlAndalus Magreb: Estudios árabes e islámicos, n², pp. 389-412.

Polinsky, Maria ; Kagan, Olga (2007) Heriatge Languages: In the 'Wild' and in the Classroom, Language and Linguistics Compass, vol.1, n5, pp. 368-395.

Serra, Josep Maria (2010) Escola, llengua i immigració a Catalunya. Algunes reflexions, Llengua, societat $i$ counicació, $\mathrm{n}^{\circ} 8, \mathrm{pp} .27-34$.

Tilmatine, Mohand (2005) La immigració amaziga a Catalunya: l'aposta identitària i ciutadana, Llengua i ús. Revista tècnica de política lingüística, n³4, pp 84-89.

Valdés, Guadalupe (2000) The teaching of heritage languages: An introduction for Slavic-teaching professionals, in Kagan, Olga ; Rifkin, Benjamin (eds.) The Learning and Teaching of Slavic Languages and Cultures, Bloomingon, Slavica, pp. 375-403.

Van Deusen-Scholl, Nelleke (2003) Toward a definition of heritage language: Sociopolitical and pedagogical considerations, Journal of Language, Identity, and Education, vol. 2, n³, pp. 211-230.

Wong Fillmore, Lily (2000) Loss of family languages: Should educators be concerned?, Theory into Practice, vol. 39, $\mathrm{n}^{\circ} 4$, pp. 203-210.

\section{NOTAS}

1. Grup d'Estudi de Llengües Amenaçades, Universitat de Barcelona (www.gela.cat)

2. Algunos lingüistas consideran el amazig un conjunto de variedades dialectales (Chaker, 1995; Múrcia, 2015) mientras que otros afirman que es un grupo de lenguas diferentes (Naït-Zerrad, 
2001; Kossmann, 2012). Aquí, siguiendo a Chaker (1995) y Múrcia (2015), hablaremos de lengua amazig.

3. Además de los 34 informantes nacidos en Marruecos, se obtuvieron 7 cuestionarios de estudiantes nacidos en España con antecedentes marroquíes (uno de los progenitores o ambos había nacido en Marruecos). No obstante, no fueron tenidos en cuenta en el estudio, porque los criterios de inclusión en la muestra global eran los enumerados en el presente apartado (nacidos fuera del Estado español). Estos criterios se establecieron teniendo en cuenta el hecho de que algunos de los estudiantes nacidos en España con antecedentes extranjeros pueden declarar como lenguas iniciales las de los países de acogida. Respecto a los siete que hemos mencionado, solo dos declaran hablar árabe o amazig. Los cinco restantes no consignan en sus respuestas ninguna de las lenguas propias de Marruecos.

4. Fabà y Torres-Pla (2020: 215) definen la lengua de identificación como aquella que muestra principalmente la identidad lingüística individual, es decir, la lengua que la persona siente emocionalmente como propia.

5. A pesar de contar con una muestra pequeña, establecemos los porcentajes, ya que consideramos que son útiles para mostrar tendencias que podrían confirmarse en estudios posteriores con muestras más amplias.

6. Els resultats escolars de les criatures que provenen de la immigració extracomunitària obtenen resultats significativament més baixos que els resultats que obtenen els alumnes autòctons.

7. [E]l fracàs seria menor entre els d'origen europeu (11,3\%) i americà $(15,5 \%)$, on la condició socioeconòmica dels primers i la proximitat a l'idioma dels segons justificarien els millors resultats. Pel conglomerat asiàtic el fracàs ascendeix al 17,8\% [...]. A la cua, amb el 19,2\% entre els alumnes, trobem els d'origen africà. En aquest darrer cas, el 15,9\% de fracàs escolar entre els alumnes marroquins és preocupant tractant-se del primer origen, especialment si tenim en compte el creixement d'aquest grup en un futur immediat (Bayona i Domingo, 2018, 11).

8. Els nanos que tenen l'amazic com a llengua materna tenen una situació semblant a la nostra [entre l'amazic i l'àrab], i això ha provocat una millor predisposició, certa simpatia, a aprendre la llengua i a utilitzar-la. [...] Actitud positiva i simpatia vers el català, perquè relacionen la situació amb l'amazic. (Manlleu, tutor d'aula d'acollida). (Bretxa et al. 2017, 106)

9. Esta presencia toma formas diversas: en algunos municipios catalanes hace tiempo que se celebra la primavera amazig, han existido instituciones dedicadas a la promoción de la lengua (como la Casa amaziga de Catalunya), hay escritores y políticos de origen amazig (Najat el Hachmi, Laila Karrouch, Salem Zenia, Chaker en Homrani o Fatima Taleb, entre otros). El Parlamento catalán, por otro lado, fue el primero en Europa, a excepción del Parlamento Europeo, en adoptar una resolución de apoyo a la identidad, la lengua y la cultura amazig (Boletín Oficial del Parlament de Catalunya de 22 de abril de 2002) (Tilmatine, 2005).

10. "les dades de la nostra mostra poden estar condicionades per la circumscripció a l'àmbit universitari, entenent que la situació sociocultural podria tenir certa influència en la transmissió de les llengües d'herència" (Barrieraas i Ferrerós, 2019: 243)

\section{RESÚMENES}

La investigación que presentamos, que se incluye en el ámbito de estudio de las lenguas de herencia y de la planificación lingüística familiar, tiene como objetivo analizar la transmisión de las lenguas de herencia árabe y amazig en Catalunya, en este caso entre los estudiantes 
universitarios de origen marroquí. La comunidad marroquí es la comunidad de origen extranjero más numerosa y más antigua en Catalunya, y gran parte de los individuos de esta comunidad tiene como primera lengua el amazig (Castellanos, 2019; Badosa i Ferrerós, 2019). Se pretende observar si hay diferencias en la transmisión del amazig, que es una lengua minorizada, respecto al árabe $\mathrm{y}$, en dicho supuesto, si esta diferencia también se manifiesta en la lengua de identificación de los hablantes. Por este motivo, y en caso de transmisión, también se analizarán los usos y las representaciones lingüísticas. Los datos presentados en este estudio se han obtenido mediante un cuestionario con preguntas semicerradas a estudiantes de origen extranjero de la Universitat de Barcelona y se han analizado cuantitativamente. No se pretende hacer una proyección sobre los estudiantes universitarios en general ni sobre la población inmigrante; se trata de una prospección que deberá permitir identificar tendencias y fenómenos interesantes que puedan guiar futuras investigaciones. A la luz de los resultados de la muestra, el artículo evidencia que las lenguas de herencia se transmiten en un porcentaje elevado y que se consignan frecuentemente como lenguas de identificación, en particular el amazig.

ÍNDICE

Palabras claves: lenguas de herencia, planificación lingüística familiar, transmisión lingüística, árabe, amazig

\section{AUTORES}

\section{MÒNICA BARRIERAS ANGÀS}

Grup d'Estudi de Llengües Amenaçades, Universitat de Barcelona

mbarrieras@ub.edu

\section{CARLA FERRERÓS PAGÈS}

Grup de Llengües, Gramàtica i Discurs, Universitat de Girona

carla.ferreros@udg.edu 